\title{
Combination therapy of fenofibrate and ursodeoxycholic acid in patients with primary biliary cirrhosis who respond incompletely to UDCA monotherapy: a meta-analysis
}

\author{
This article was published in the following Dove Press journal: \\ Drug Design, Development and Therapy \\ 25 May 2015 \\ Number of times this article has been viewed
}

Yan Zhang',2,*
Sainan Li',
Lei He'
Fan Wang'
Kan Chen'
Jingjing Li'
Tong Liu'
Yuanyuan Zheng'
Jianrong Wang ${ }^{1,3}$
Wenxia Lu',3
Yuqing Zhou ${ }^{1,4}$
Qin Yin',4
Yujing Xia'
Yingqun Zhou'
Jie Lu'
Chuanyong Guo'
'Department of Gastroenterology,
Shanghai Tenth People's Hospital,
Tongji University School of Medicine,
Shanghai, ${ }^{2}$ Sir Run Run Shaw Hospital,
Zhejiang University School of
Medicine, Hangzhou, ${ }^{3}$ The First Clinical
Medical College of Nanjing Medical
University, Nanjing, ${ }^{4}$ The First Affiliated
Hospital of Soochow University,
Suzhou, People's Republic of China
*These authors contributed equally
to this work
to this work

Correspondences: Chuanyong Guo; Jie Lu Department of Gastroenterology, Shanghai Tenth People's Hospital, Tongii University School of Medicine, Shanghai 200072, People's Republic of China

Email guochuanyong@hotmail.com; kennisren@hotmail.com

\begin{abstract}
Background: Although the effectiveness of treatment with ursodeoxycholic acid (UDCA) and fenofibrate for primary biliary cirrhosis (PBC) has been suggested by small trials, a systematic review to summarize the evidence has not yet been carried out.

Methods: A meta-analysis of all long-term randomized controlled trials comparing the combination of UDCA and fenofibrate with UDCA monotherapy was performed via electronic searches.

Results: Six trials, which included 84 patients, were assessed. Combination therapy with UDCA and fenofibrate was more effective than UDCA monotherapy in improving alkaline phosphatase (mean difference [MD]: $-90.44 \mathrm{IU} / \mathrm{L} ; 95 \%$ confidence interval [CI]: -119.95 to $-60.92 ; P<0.00001$ ), gamma-glutamyl transferase (MD: $-61.58 \mathrm{IU} / \mathrm{L} ; 95 \% \mathrm{CI}:-122.80$ to $-0.35 ; P=0.05$ ), immunoglobulin M (MD: $-38.45 \mathrm{mg} / \mathrm{dL} ; 95 \% \mathrm{CI}:-64.38$ to $-12.51 ; P=0.004)$, and triglycerides (MD: $-0.41 \mathrm{mg} / \mathrm{dL} ; 95 \% \mathrm{CI}:-0.82$ to $-0.01 ; P=0.05$ ). However, their effects on pruritus (odds ratio [OR]: $0.39 ; 95 \% \mathrm{CI}: 0.09-1.78 ; P=0.23$ ), total bilirubin (MD: $-0.05 \mathrm{mg} / \mathrm{dL}$; 95\% CI: -0.21 to $0.12 ; P=0.58$ ), and alanine aminotransferase (MD: $-3.31 \mathrm{IU} / \mathrm{L} ; 95 \% \mathrm{CI}:-14.60$ to $7.97 ; P=0.56$ ) did not differ significantly. This meta-analysis revealed no significant differences in the incidence of adverse events (OR: $0.21 ; 95 \% \mathrm{CI}$ : $0.03-1.25 ; P=0.09$ ) between patients treated with combination therapy and those treated with monotherapy.

Conclusion: In this meta-analysis, combination therapy with UDCA and fenofibrate was more effective in reducing alkaline phosphatase than UDCA monotherapy, but it did not improve clinical symptoms. There did not appear to be an increase in adverse events with combination therapy.
\end{abstract}

Keywords: randomized controlled trials, combination therapy, monotherapy, fibrates, odds ratio, risk difference

\section{Introduction}

Primary biliary cirrhosis (PBC) is a chronic progressive inflammatory autoimmunemediated cholestatic disease that mainly occurs in elderly women and is characterized by progressive inflammatory destruction of the interlobular bile duct, and the subsequent development of liver fibrosis and cirrhosis, eventually leading to liver failure. ${ }^{1}$ The etiology and exact pathogenesis of PBC are still not entirely clear, and may be related to genetic factors, viral and bacterial infections, autoimmune status, and environmental factors. ${ }^{2}$ Ursodeoxycholic acid (UDCA) is currently the only United States Food and Drug Administration (FDA)-approved and the 2009 American Association for the Study of Liver Diseases Practice Guidelines-recommended drug for the treatment of PBC. ${ }^{3}$ UDCA therapy for PBC is very safe, and it can improve 
clinical symptoms and liver function abnormality indicators, delay histologic progression, improve quality of life, prolong survival, and prolong transplant-free survival. ${ }^{4}$ However, up to $40 \%$ of patients treated with UDCA monotherapy have a suboptimal response (no significant decrease in alkaline phosphatase [ALP], alanine aminotransferase [ALT], or immunoglobulin [Ig]M), and 10\% will subsequently die or require liver transplantation, indicating a clear need for additional therapies. ${ }^{4,5}$

Fenofibrate, a peroxisome proliferator-activated receptor (PPAR)- $\alpha$-selective agonist, is a fibric acid derivative that is US FDA approved and clinically used for the treatment of hypercholesterolemia and hypertriglyceridemia. ${ }^{6-8}$ Recent pilot studies in patients with PBC refractory to UDCA monotherapy demonstrated that fenofibrate can also improve biochemical and immunological indicators to normal levels, and significantly improve clinical symptoms without side effects. ${ }^{9,10}$ Therefore, we conducted this meta-analysis to evaluate the clinical outcomes of the combination of UDCA and fenofibrate compared with UDCA monotherapy in patients with $\mathrm{PBC}$.

\section{Methods}

\section{Study identification}

Relevant studies were searched and selected by searching the PubMed, the Cochrane Library, Embase, and CINAHL databases, as well as the Science Citation Index (updated to September 2013) using the search terms "ursodeoxycholic acid", "fenofibrate", "PBC", and "randomized controlled trial". We also carried out a full manual search of all review articles, and retrieved original studies and abstracts. ${ }^{11}$

\section{Inclusion criteria}

The studies included in this study fit the following three criteria. First, in terms of study design, randomized controlled trials (RCTs) comparing combination therapy with UDCA/ fenofibrate and monotherapy with UDCA were included. Second, with respect to study population, the diagnosis of PBC was made on the basis of any two of the following criteria: 1) positive test for antimitochondrial antibody; 2) biochemical evidence of cholestasis; and 3) liver biopsy compatible with the diagnosis. ${ }^{12}$ Parés et al ${ }^{4}$ suggested that patients who show a drop of $>40 \%$ in serum ALP levels from baseline or normalization have similar survival rates when compared with the general age- and sex-matched population. Therefore, we defined a complete biochemical response to treatment as an ALP level decrease of more than $40 \%$ of the baseline values or normal range after 1 year of UDCA treatment. Third, duplicated publications were excluded and no language or date limitations were imposed. There was also no limitation on the form of publication.

\section{Data extraction}

Data were independently collected from each study by two researchers (Yan Zhang and Sainan Li) to confirm the accuracy of the data. The following data were extracted from each included article: name of the first author, year of publication, number of patients, daily dose of oral therapy, duration of treatment, method used to deal with missing data, liver biochemistry, symptoms, death, and adverse events.

\section{Assessment of risk of bias in included studies}

There are a lot of tools available to assess the methodological quality of clinical trials. According to the Cochrane Handbook 5.0.2, the Cochrane Collaboration recommends a specific tool for assessing the risk of bias in each included study. ${ }^{13-15}$ This consists of a description of and a judgment for each entry in a "Risk of bias" table, where each entry addresses a specific feature of the study. The judgment for each entry involves answering a question, with answers "Yes" indicating low risk of bias, "No" indicating high risk of bias, and "Unclear" indicating either a lack of information or uncertainty over the potential for bias. For parallel group trials, the features of interest in a standard "Risk of bias" table of a Cochrane review are allocation sequence generation, allocation concealment, blinding, incomplete outcome data, selective outcome reporting, and other potential sources of bias (Table 1).

\section{Statistical analyses}

The data collected were processed by RevMan 5.2 (The Nordic Cochrane Centre, Copenhagen, Denmark; The Cochrane Collaboration, 2012). We calculated the odds ratio (OR) and/ or risk difference for dichotomous outcomes, and the mean difference (MD) for continuous outcomes, all with $95 \%$ confidence intervals (CIs). We tested heterogeneity using the $\chi^{2}$ test and the $I^{2}$ test, and a $P$-value of $<0.10$ or an $I^{2}$ value of $>50 \%$ was considered as substantial heterogeneity. A fixed-effects model was used when the heterogeneity test showed a $P$-value of $>0.10$ and an $I^{2}$ value of $<50 \%$; otherwise, a random-effect model was used. We also constructed funnel plots to evaluate the presence of publication bias.

\section{Results}

\section{Descriptive and qualitative assessments}

This meta-analysis included 84 patients (Figure 1). The general characteristics of the six trials are shown in Table 2. 
Table I Criteria used to assess the risk of bias in the included studies

Trials assessed as having a "low risk of bias" in all the specified individual domains were considered as "trials with low risk of bias". Trials assessed as having an "uncertain risk of bias" or a "high risk of bias" in one or more of the specified individual domains were considered "trials with high risk of bias".

\section{Allocation sequence generation}

- Low risk of bias: sequence generation was achieved using computer random number generation or a random number table. Drawing lots, tossing a coin, shuffling cards, and throwing dice are adequate if performed by an independent adjudicator.

- Uncertain risk of bias: the trial is described as randomized, but the method of sequence generation was not specified.

- High risk of bias: the sequence generation method is not, or may not be, random. Quasi-randomized studies - those using dates, names, or admittance numbers in order to allocate patients - are inadequate and will be excluded for the assessment of benefits, but not for harms.

\section{Allocation concealment}

- Low risk of bias:allocation was controlled by a central and independent randomization unit, sequentially numbered, and in opaque and sealed envelopes or similar, so that intervention allocations could not have been foreseen in advance of, or during, enrolment.

- Uncertain risk of bias: the trial was described as randomized, but the method used to conceal the allocation was not described, so that intervention allocations may have been foreseen in advance of, or during, enrolment.

- High risk of bias: if the allocation sequence was known to the investigators who assigned patients or if the study was quasi-randomized. Quasi-randomized studies will be excluded for the assessment of benefits, but not for harms.

Blinding

- Low risk of bias: the trial was described as blinded; the parties that were blinded, and the method of blinding were described, so that knowledge of allocation was adequately prevented during the trial.

- Uncertain risk of bias: the trial was described as blind, but the method of blinding was not described, so that knowledge of allocation was possible during the trial.

- High risk of bias: the trial was not blinded, so that the allocation was known during the trial.

Incomplete outcome data

- Low risk of bias: the numbers and reasons for dropouts and withdrawals in all intervention groups were described, or if it was specified, that there were no dropouts or withdrawals.

- Uncertain risk of bias: the report gave the impression that there had been no dropouts or withdrawals, but this was not specifically stated.

- High risk of bias: the number or reasons for dropouts and withdrawals were not described.

Selective outcome reporting

- Low risk of bias: predefined or clinically relevant and reasonably expected outcomes are reported on.

- Uncertain risk of bias: not all predefined or clinically relevant and reasonably expected outcomes are reported on, or they are not reported fully, or it is unclear whether data on these outcomes were recorded or not.

- High risk of bias: one or more clinically relevant and reasonably expected outcomes were not reported on; data on these outcomes were likely to have been recorded.

Other bias

- Low risk of bias: the trial appears to be free of other components that could increase risk of bias.

- Uncertain risk of bias: the trial may or may not be free of other components that could increase the risk of bias.

- High risk of bias: there are other factors in the trial that could increase the risk of bias - eg, for-profit involvement, the authors have conducted trials on the same topic, etc.

The mean ages ranged from 51 to 61 years and the mean follow-up intervals ranged from 3 to 24 months. The daily dose of UDCA ranged from 600 to $900 \mathrm{mg} /$ day, and the daily dose of fenofibrate was 134-200 mg/day. Not all studies were published as full-text articles. ${ }^{16-21}$ One trial was published as an abstract and one as a letter to the editor. ${ }^{17,18}$ The results of the meta-analysis are shown in Table 3. The six included trials were evaluated with the help of the "assessing risk of bias" table and the outcome is summarized in Figure 2. Risk of bias was assessed according to six components: allocation sequence generation, allocation concealment, blinding, handling of incomplete outcome data, selective outcome reporting, and other potential sources of bias. All trials were assessed as having a high risk of bias. Our statistical analyses are, therefore, based only on trials with a high risk of bias (Figure 3). Figure 4 shows the funnel plots of the meta-analysis. The funnel plots for clinical events showed slight asymmetry, suggesting possible publication bias.

\section{Evaluation of the effects of therapy}

Four studies ${ }^{17,19-21}$ reported the clinical outcome of the treatments on patients' symptoms, and found improvement in pruritus. The combination therapy significantly improved liver function and reduced serum ALP levels in all the included studies. Five studies $^{16,17,19-21}$ also reported adverse effects (heartburn, nausea, aggravated pruritus, and fatigue) and yet, no serious adverse events were reported. There were no deaths in the five studies..$^{16,17,19-21}$

\section{Meta-analysis}

\section{ALP levels}

Six trials, ${ }^{16-21}$ which included 84 patients, reported data regarding this endpoint. Combination therapy with UDCA 


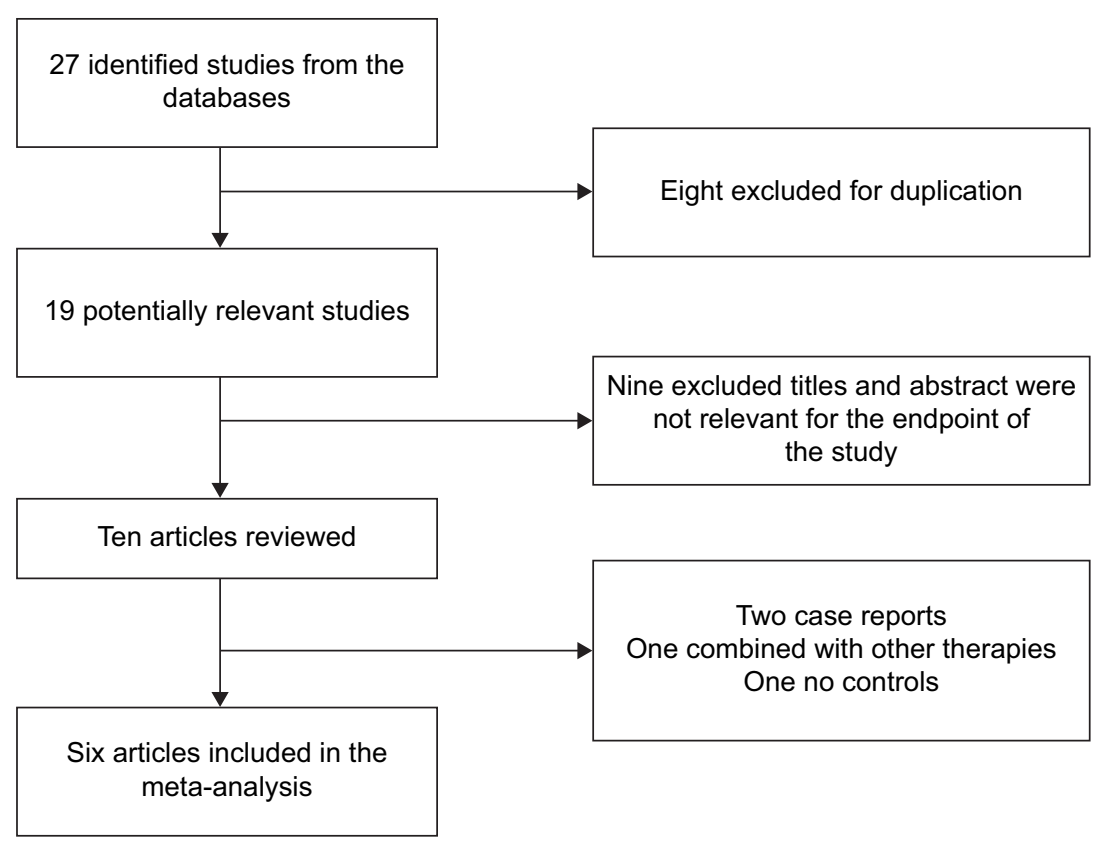

Figure I Flow diagram of the studies included in the meta-analysis.

Table 2 Baseline characteristics of the trials included in the meta-analysis

\begin{tabular}{|c|c|c|c|c|c|c|c|}
\hline \multirow[t]{2}{*}{ References } & \multirow{2}{*}{$\begin{array}{l}\text { Mean age } \\
\text { (years) }\end{array}$} & \multirow{2}{*}{$\begin{array}{l}\text { Patients } \\
\text { (n) }\end{array}$} & \multirow{2}{*}{$\begin{array}{l}\text { UDCA dose } \\
\text { (mg/day) }\end{array}$} & \multirow{2}{*}{$\begin{array}{l}\text { Fenofibrate dose } \\
\text { (mg/day) }\end{array}$} & \multicolumn{2}{|c|}{ Duration of treatment } & \multirow{2}{*}{$\begin{array}{l}\text { Publication } \\
\text { type }\end{array}$} \\
\hline & & & & & UDCA & COM & \\
\hline Ohira et al ${ }^{17}$ & 61 & 7 & $600-900$ & $150-200$ & 8 years & 6 months & Letter \\
\hline Dohmen et $\mathrm{al}^{16}$ & 53 & 9 & 600 & $100-150$ & 6 months & 3 months & Full text \\
\hline Walker et al ${ }^{18}$ & 55 & 16 & $600-900$ & $134-200$ & 23 months & 23 months & Letter \\
\hline Liberopoulos et al ${ }^{19}$ & 57 & 10 & 600 & 200 & 8 months & 2 months & Full text \\
\hline Levy et $\mathrm{a}^{20}$ & 56 & 20 & $600-900$ & 160 & 12 months & 12 months & Full text \\
\hline Han et $\mathrm{al}^{21}$ & 51 & 22 & $600-900$ & 200 & 18 months & 6 months & Full text \\
\hline
\end{tabular}

Abbreviations: $n$, number; UDCA, ursodeoxycholic acid; COM, combination treatment.

Table 3 Meta-analysis of clinical events and biochemical parameter changes in the included studies

\begin{tabular}{|c|c|c|c|c|c|}
\hline Outcome title & $\begin{array}{l}\text { Number of } \\
\text { studies }\end{array}$ & $\begin{array}{l}\text { Number of } \\
\text { participants }\end{array}$ & Statistical method & Effect size & $P$-value \\
\hline Pruritus & 4 & 59 & $\begin{array}{l}\text { Odds ratio } \\
(\mathrm{M}-\mathrm{H} \text {, fixed, } 95 \% \mathrm{Cl})\end{array}$ & $0.39(0.09-1.78)$ & 0.23 \\
\hline Adverse events & 5 & 68 & $\begin{array}{l}\text { Odds ratio } \\
(\mathrm{M}-\mathrm{H} \text {, fixed, } 95 \% \mathrm{Cl})\end{array}$ & $0.21(0.03-1.25)$ & 0.09 \\
\hline $\begin{array}{l}\text { Alkaline } \\
\text { phosphatase }\end{array}$ & 6 & 84 & $\begin{array}{l}\text { Mean difference } \\
(\mathrm{IV}, \text { fixed, } 95 \% \mathrm{Cl})\end{array}$ & $-90.44(-119.95$ to -60.92$)$ & $<0.00001$ \\
\hline $\begin{array}{l}\text { Gamma-glutamyl } \\
\text { transferase }\end{array}$ & 4 & 48 & $\begin{array}{l}\text { Mean difference } \\
\text { (IV, fixed, } 95 \% \mathrm{Cl} \text { ) }\end{array}$ & $-61.58(-122.80$ to -0.35$)$ & 0.05 \\
\hline Immunoglobulin M & 5 & 62 & $\begin{array}{l}\text { Mean difference } \\
\text { (IV, fixed, } 95 \% \mathrm{Cl} \text { ) }\end{array}$ & $-38.45(-64.38$ to $-12.5 \mid)$ & 0.004 \\
\hline $\begin{array}{l}\text { Alanine } \\
\text { aminotransferase }\end{array}$ & 4 & 61 & $\begin{array}{l}\text { Mean difference } \\
\text { (IV, fixed, } 95 \% \mathrm{Cl} \text { ) }\end{array}$ & -3.31 ( -14.60 to 7.97$)$ & 0.56 \\
\hline Total bilirubin & 4 & 61 & $\begin{array}{l}\text { Mean difference } \\
\text { (IV, fixed, } 95 \% \mathrm{Cl} \text { ) }\end{array}$ & $-0.05(-0.21$ to 0.12$)$ & 0.58 \\
\hline Triglycerides & 4 & 61 & $\begin{array}{l}\text { Mean difference } \\
\text { (IV, fixed, } 95 \% \mathrm{Cl} \text { ) }\end{array}$ & $-0.41(-0.82$ to -0.01$)$ & 0.05 \\
\hline
\end{tabular}

Abbreviations: $\mathrm{M}-\mathrm{H}$, Mantel-Haenszel; $\mathrm{Cl}$, confidence interval; IV, inverse-variance. 


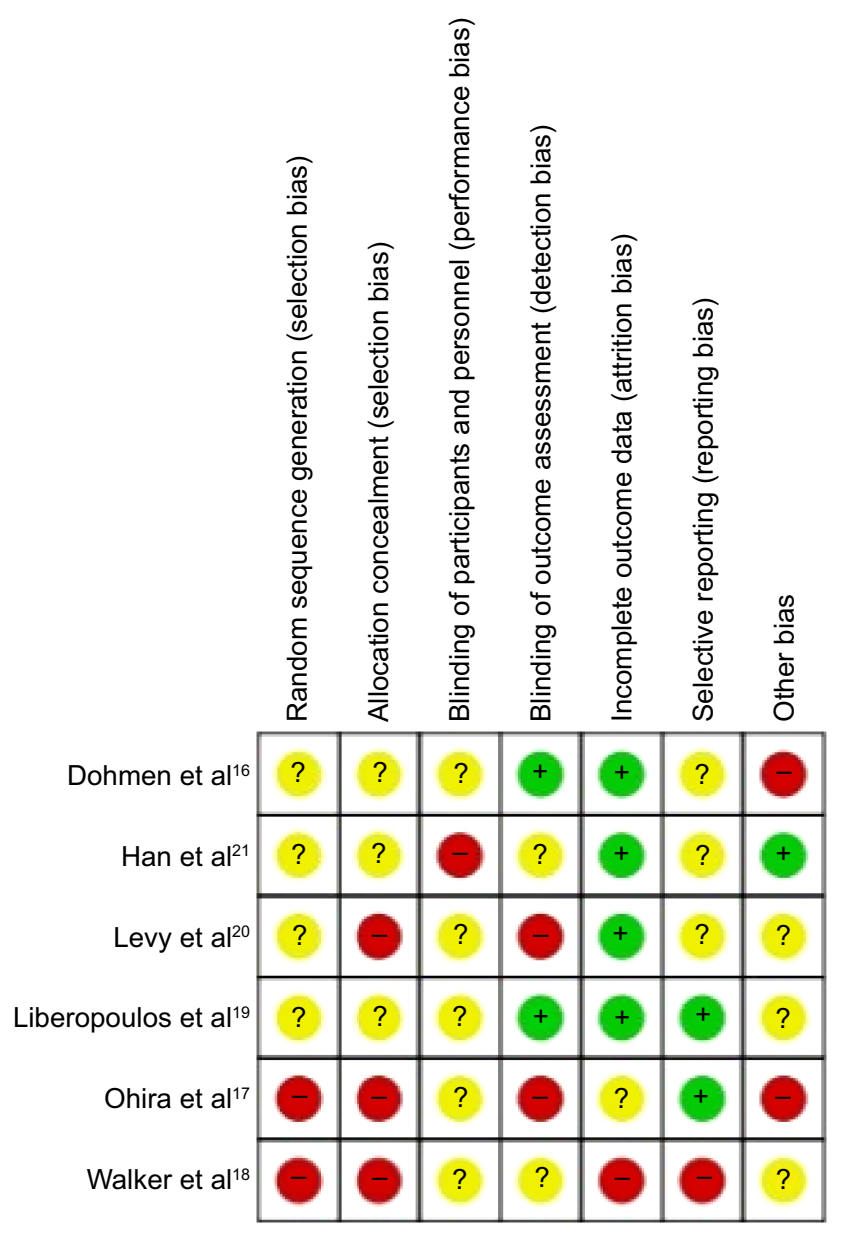

$$
+ \text { Low risk of bias ? Unclear } \quad \text { - High risk of bias }
$$

Figure 2 Risk of bias in the included studies. and fenofibrate was more effective than UDCA monotherapy in decreasing serum ALP (MD: $-90.44 \mathrm{IU} / \mathrm{L} ; 95 \%$ CI: -119.95 to $-60.92 ; P<0.00001$; Figure 5). There was no significant heterogeneity $\left(P=0.11 ; I^{2}=45 \%\right)$.

\section{Pruritus}

Four trials, ${ }^{17,19-21}$ which included 59 patients, reported data regarding this endpoint. Symptoms improved in one of 53 patients in the monotherapy groups and in one of 55 patients in the combination therapy groups. There was no significant heterogeneity $\left(P=0.77 ; I^{2}=0 \%\right)$ and no significant differences between the groups (OR: 0.39 ; $95 \%$ CI: $0.09-1.78$; $P=0.23$; Figure 6).

\section{Gamma-glutamyl transferase $(\gamma-\mathrm{GT})$ levels}

Four trials, ${ }^{16,17,20,21}$ which included 48 patients, reported data regarding this endpoint. The combination therapy significantly decreased serum $\gamma$-GT compared with UDCA monotherapy (MD: $-61.58 \mathrm{IU} / \mathrm{L} ; 95 \% \mathrm{CI}:-122.80$ to -0.35 ; $P=0.05$; Figure 7). There was no significant heterogeneity $\left(P=0.38 ; I^{2}=2 \%\right)$.

\section{IgM levels}

Five trials, ${ }^{16-20}$ which included 62 patients, reported data regarding this endpoint. The combination therapy significantly decreased serum IgM levels compared with UDCA monotherapy (MD: $-38.45 \mathrm{mg} / \mathrm{dL}$; $95 \% \mathrm{CI}:-64.38$ to

Random sequence generation (selection bias)

Allocation concealment (selection bias)

Blinding of participants and personnel (performance bias)

Blinding of outcome assessment (detection bias)

Incomplete outcome data (attrition bias)

Selective reporting (reporting bias)
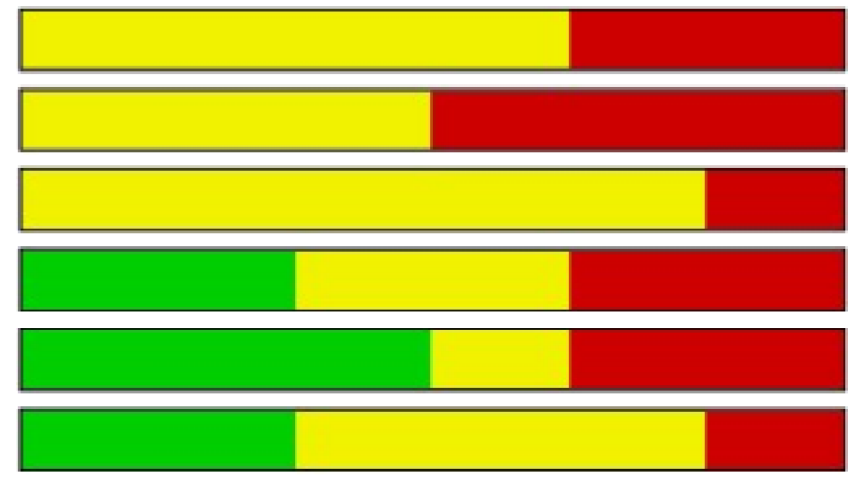

Other bias

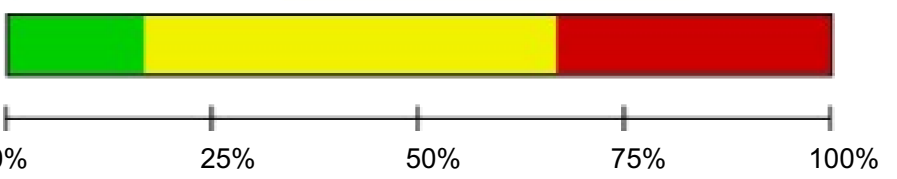

Low risk of bias

Unclear risk of bias

High risk of bias

Figure 3 Risk of bias graph: review of the authors' judgments regarding each risk of bias item presented as percentages across all included studies. 

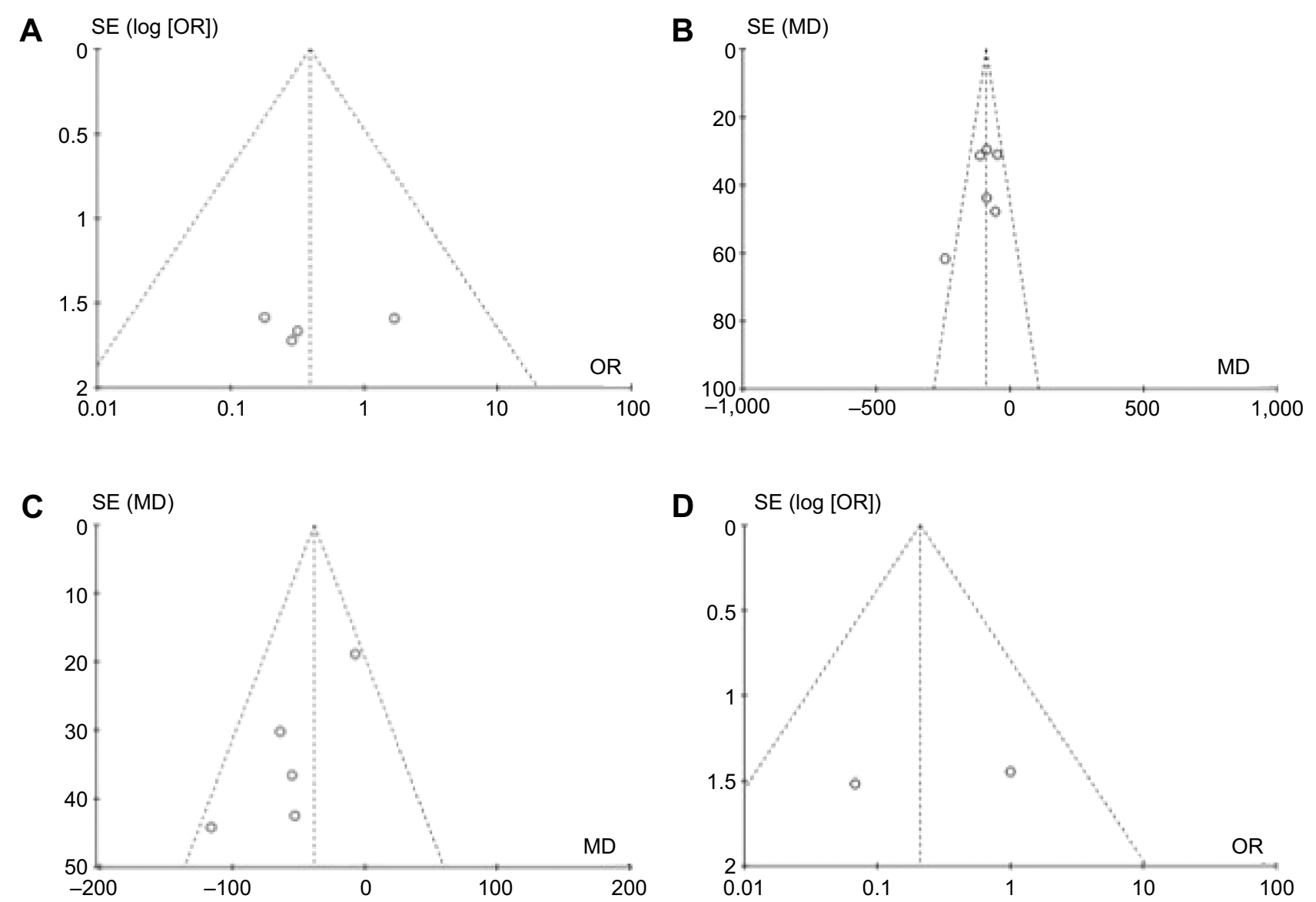

Figure 4 Funnel plots for the meta-analysis.

Notes: (A) Symptoms of pruritus. (B) Alkaline phosphatase. (C) Immunoglobulin M. (D) Adverse events.

Abbreviations: SE, standard error; OR, odds ratio; MD, mean difference.

$-12.51 ; P=0.004 ;$ Figure 8$)$. There was no significant heterogeneity $\left(P=0.14 ; I^{2}=42 \%\right)$.

\section{ALT levels}

Four trials, ${ }^{16,19-21}$ which included 61 patients, reported data regarding this endpoint. The combination therapy compared with monotherapy had no significant effect on serum ALT levels (MD: $-3.31 \mathrm{IU} / \mathrm{L} ; 95 \% \mathrm{CI}:-14.60$ to $7.97 ; P=0.56$;
Figure 9). There was no significant heterogeneity $(P=0.99$; $\left.r^{2}=0 \%\right)$.

\section{Triglyceride levels}

Four trials, ${ }^{16,19-21}$ which included 61 patients, reported data regarding this endpoint. Combination therapy significantly decreased triglyceride levels compared with UDCA monotherapy (MD: $-0.41 \mathrm{mg} / \mathrm{dL} ; 95 \% \mathrm{CI}:-0.82$ to -0.01 ;

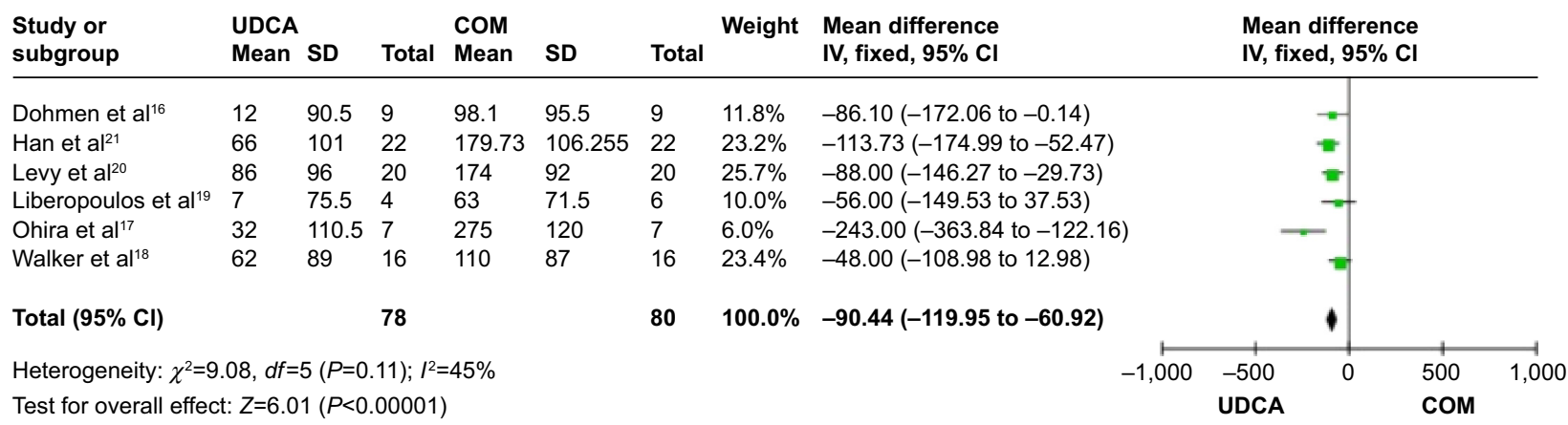

Figure 5 ALP levels in PBC patients treated with monotherapy versus COM.

Abbreviations: ALP, alkaline phosphatase; UDCA, ursodeoxycholic acid; COM, combination therapy; SD, standard deviation; IV, inverse-variance; CI, confidence interval; $d f$, degrees of freedom; PBC, primary biliary cirrhosis. 


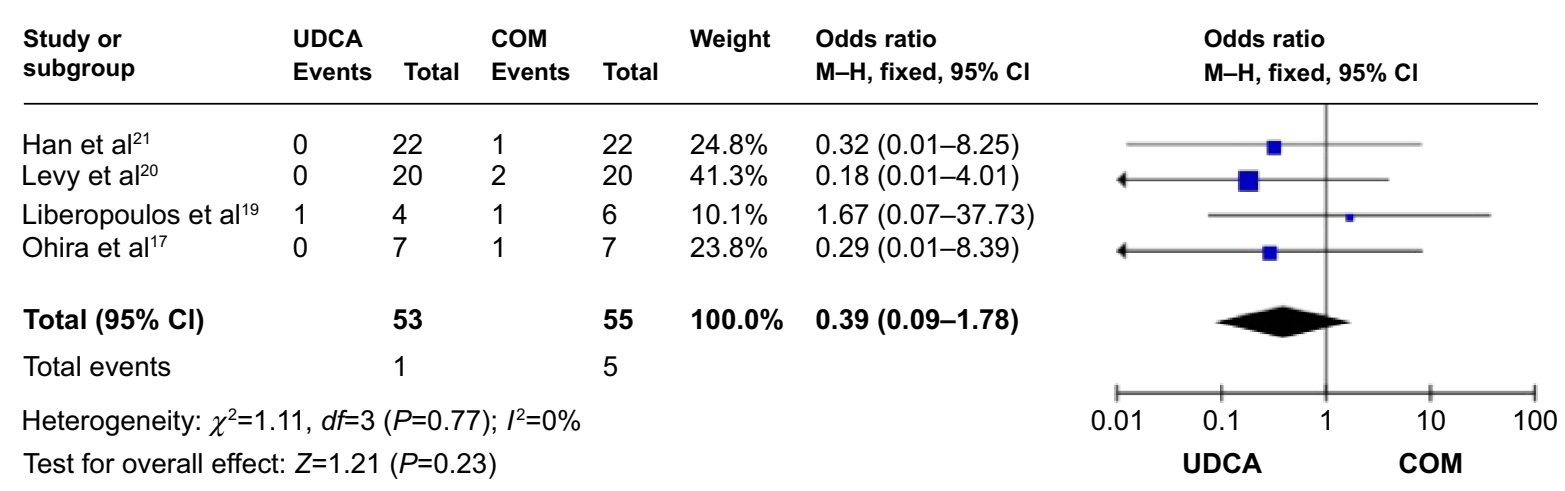

Figure 6 Effects of monotherapy versus COM on pruritus in patients with PBC.

Abbreviations: UDCA, ursodeoxycholic acid; COM, combination therapy; M-H, Mantel-Haenszel; $\mathrm{Cl}$, confidence interval; df, degrees of freedom; PBC, primary biliary cirrhosis.

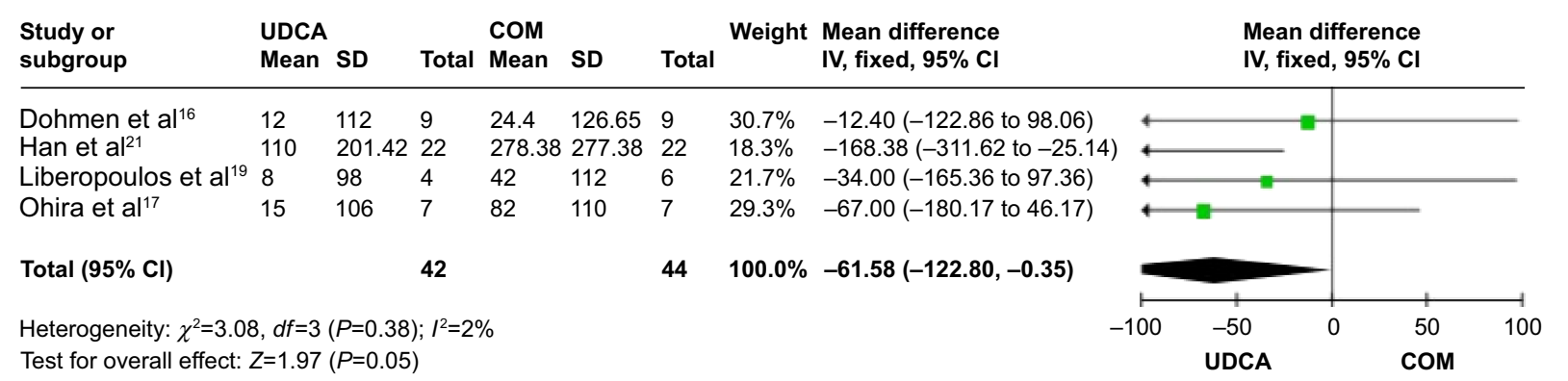

Figure $7 \gamma$-GT levels in PBC patients treated with monotherapy versus COM.

Abbreviations: UDCA, ursodeoxycholic acid; COM, combination therapy; SD, standard deviation; IV, inverse-variance; Cl, confidence interval; df, degrees of freedom; $\gamma$-GT, gamma-glutamyl transferase; PBC, primary biliary cirrhosis.

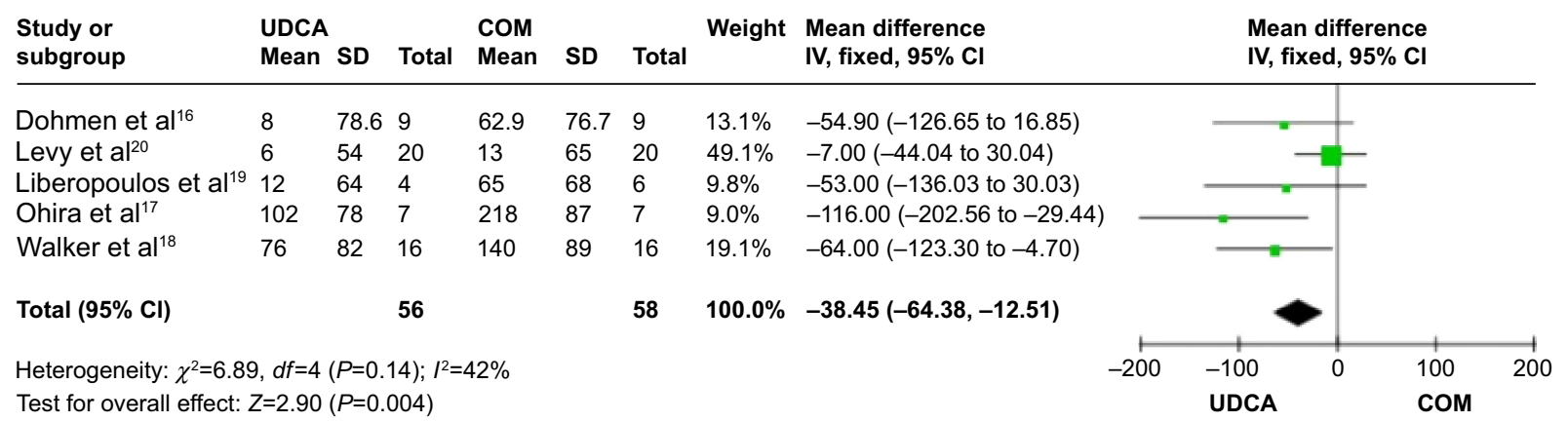

Figure 8 IgM levels in PBC patients treated with monotherapy versus COM.

Abbreviations: UDCA, ursodeoxycholic acid; COM, combination therapy; SD, standard deviation; IV, inverse-variance; Cl, confidence interval; df, degrees of freedom; IgM, immunoglobulin M; PBC, primary biliary cirrhosis.

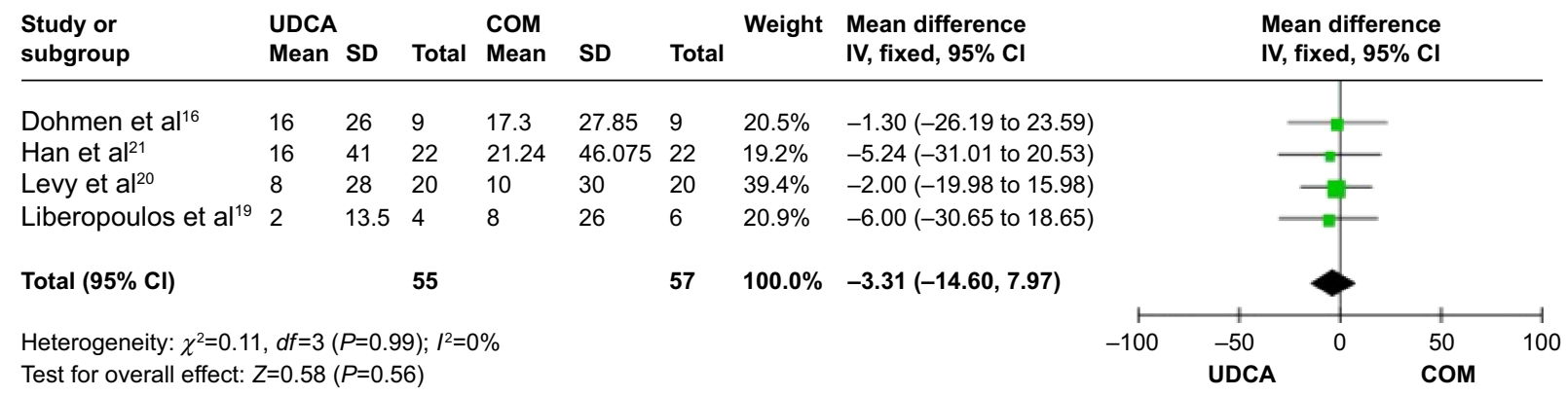

Figure 9 ALT levels in PBC patients treated with monotherapy versus COM.

Abbreviations: UDCA, ursodeoxycholic acid; COM, combination therapy; SD, standard deviation; IV, inverse-variance; Cl, confidence interval; $d f$, degrees of freedom; ALT, alanine aminotransferase; PBC, primary biliary cirrhosis. 


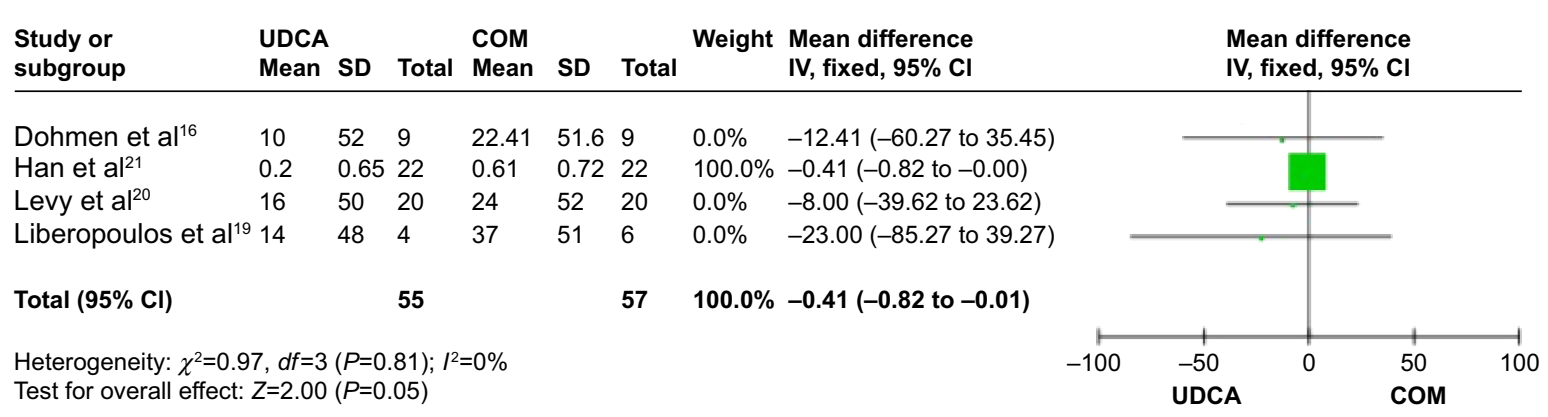

Figure 10 Triglyceride levels in PBC patients treated with monotherapy versus COM. Abbreviations: UDCA, ursodeoxycholic acid; COM, combination therapy; SD, standard deviation; IV, inverse-variance; Cl, confidence interval; df, degrees of freedom; PBC, primary biliary cirrhosis.

$P=0.05$; Figure 10$)$. There was no significant heterogeneity $\left(P=0.81 ; I^{2}=0 \%\right)$.

\section{Total bilirubin}

Four trials, ${ }^{16,19-21}$ which included 61 patients, reported data regarding this endpoint. There was no significant heterogeneity $\left(P=0.18 ; I^{2}=39 \%\right)$, and there were no significant differences between the groups (MD: $-0.05 \mathrm{IU} / \mathrm{L} ; 95 \% \mathrm{CI}:-0.21$ to $0.12 ; P=0.58$; Figure 11 ).

\section{Adverse events}

Five trials, ${ }^{16,17,19-21}$ which included 68 patients, reported data regarding this endpoint. The incidence of adverse events was one of 62 patients in the monotherapy groups and six of 64 patients in the combination therapy groups. There was no significant heterogeneity $\left(P=0.19 ; I^{2}=41 \%\right)$, and there were no significant differences between the groups (OR: 0.21 ; 95\% CI: 0.03-1.25; $P=0.09$; Figure 12).

\section{Discussion}

$\mathrm{PBC}$ is a chronic autoimmune cholestatic liver disease, and it can cause progressive liver fibrosis and eventually liver failure. ${ }^{22,23}$ Fenofibrate and bezafibrate are members of the fibrate class of hypolipidemic agents used clinically to treat hypertriglyceridemia and mixed hyperlipidemia. ${ }^{24}$ Unlike bezafibrate, fenofibrate is effective when taken only once a day, as it has a longer half-life (18 hours), and it reduces total cholesterol, similar to the effects of statin drugs. ${ }^{25}$ The activity of fenofibrate toward the PPAR is strong when compared with that of bezafibrate. ${ }^{26}$

Although many observational studies have been published, the mechanism by which fibrates reduce biochemical markers of cholestasis, and whether fibrate therapy improves survival in patients with these disorders, remain unclear. There are some hypotheses that have been postulated: ${ }^{27-30}$ 1) As PPAR- $\alpha$ agonists, fibrates improve the homeostasis of lipid concentrations in serum through PPAR systems. PPAR also acts to control inflammation through the regulation of leukotriene B4 by suppressing nuclear factor-kappa $B$ systems and activating I- $\kappa \mathrm{B}$ systems; fibrates then indirectly affect inflammation. 2) Fibrates induce $M D R 3$ gene expression and upregulate P-glycoprotein expression, thus facilitating the production of biliary phospholipids. This, in turn, results in a reduction in the cytotoxic effects of these phospholipids on the biliary epithelia. However, the mechanism of action of UDCA is different. The main effects

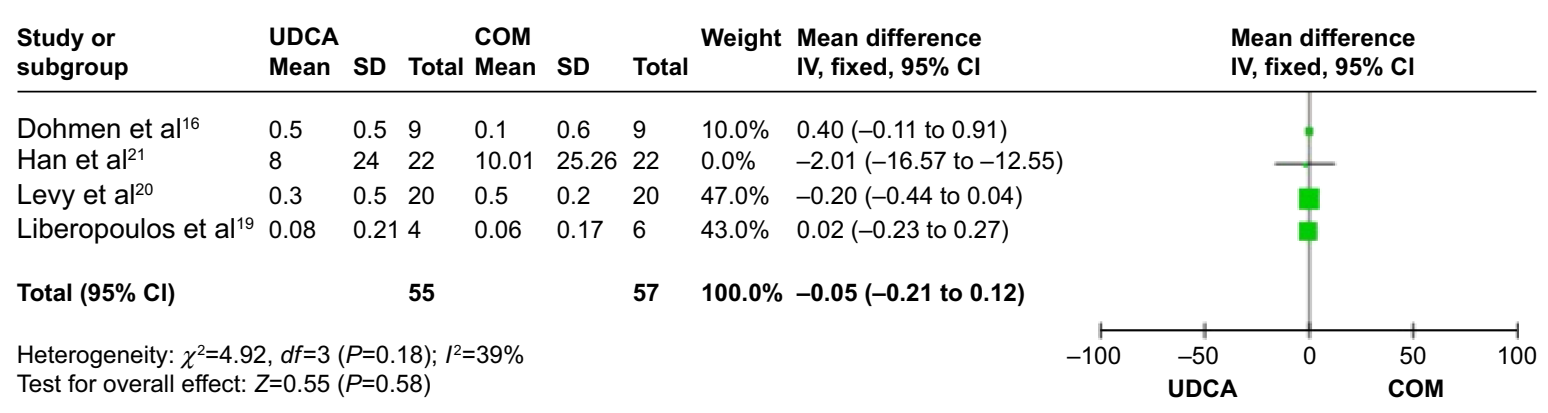

Figure II Total bilirubin levels in PBC patients treated with monotherapy versus COM.

Abbreviations: UDCA, ursodeoxycholic acid; COM, combination therapy; SD, standard deviation; IV, inverse-variance; Cl, confidence interval; df, degrees of freedom; PBC, primary biliary cirrhosis. 


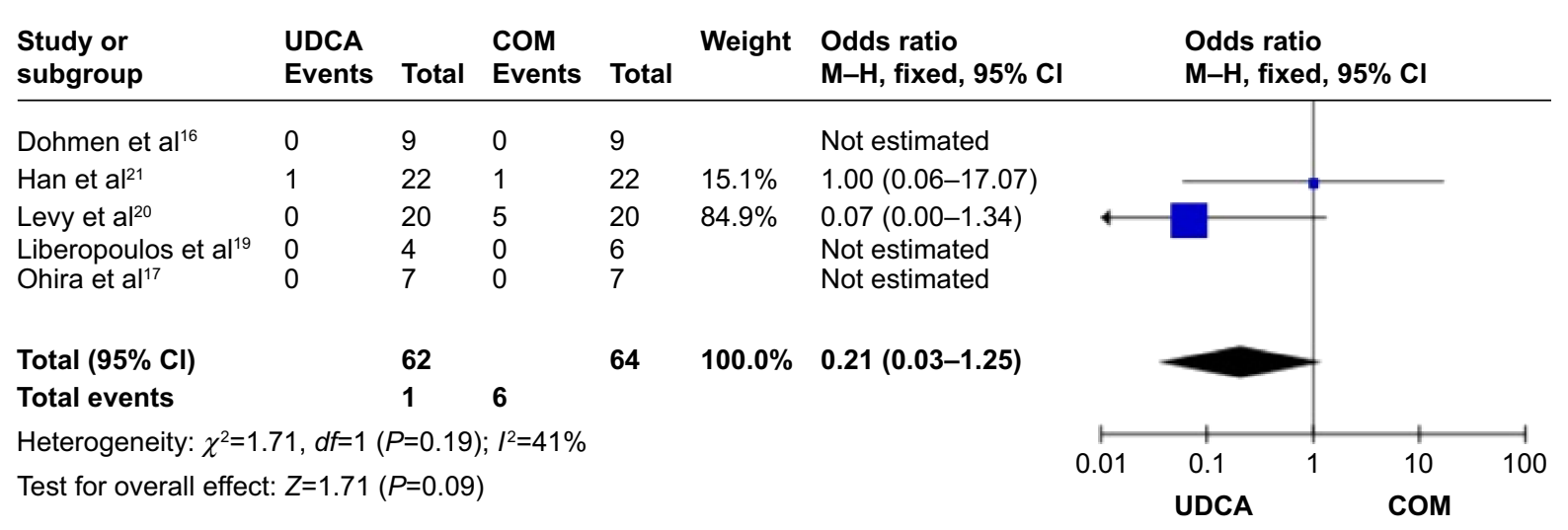

Figure 12 Adverse events in $\mathrm{PBC}$ patients treated with monotherapy versus COM.

Abbreviations: UDCA, ursodeoxycholic acid; COM, combination therapy; $\mathrm{M}-\mathrm{H}$, Mantel-Haenszel; Cl, confidence interval; df, degrees of freedom; PBC, primary biliary cirrhosis.

of UDCA are to improve the balance between toxic and nontoxic hydrophobic bile acids, and to activate the secretion of bile acids, phospholipids, and cholesterol. Thus, it does not overlap with the fibrate-affected systems, and the combination therapy of fenofibrate and UDCA may be more effective than UDCA monotherapy.

There are some limitations in this study. All six trials included in this meta-analysis had a small sample size, with an average of $14 \mathrm{PBC}$ patients, which means that there is less chance of detecting a small, but true effect as statistically significant. ${ }^{14}$ The risk of random error would be higher when data are obtained from a small sample size; thus, sample sizes need to be large enough in order to reduce the risk of random error and increase the chance of observing a true intervention effect. ${ }^{11,31,32}$ Also, we did not achieve pathological results when comparing the histologic changes before and after combination therapy. Thus, we would recommend larger controlled multicenter studies to further evaluate the clinical and laboratory outcomes of this combination therapy.

\section{Conclusion}

In summary, combination therapy with UDCA and fenofibrate provided satisfactory clinical outcomes, which may be a promising alternative for $\mathrm{PBC}$ patients refractory to UDCA. We suggest that more animal research about the pathogenesis of autoimmune liver diseases needs to be done to develop more specific and sensitive immunological parameters and genetic diagnostic techniques, ${ }^{33-38}$ which would be significant to the early diagnosis and prognostic evaluation of PBC.

\section{Acknowledgments}

This study was supported by the National Natural Science Foundation of China (grant number: 81270515), the Chinese Foundation for Hepatitis Prevention and Control (grant numbers: WBN20100021 and CFHPC20131011), and the Shanghai Municipal Health Bureau Foundation (grant numbers: 2011287 and 2012107).

\section{Author contributions}

All of the authors conceived the study, performed the literature search, quality assessment and performed the statistical analysis. All of the authors were involved in manuscript writing and preparation. All of the authors have read and approved of the final manuscript.

\section{Disclosure}

The authors report no conflicts of interest in this work.

\section{References}

1. Prince MI, James OF. The epidemiology of primary biliary cirrhosis. Clin Liver Dis. 2003;7(4):795-819.

2. Poupon R. Primary biliary cirrhosis: a 2010 update. J Hepatol. 2010; 52(5):745-758.

3. Ikegami T, Matsuzaki Y. Ursodeoxycholic acid: mechanism of action and novel clinical applications. Hepatol Res. 2008;38(2):123-131.

4. Parés A, Caballería L, Rodés J. Excellent long-term survival in patients with primary biliary cirrhosis and biochemical response to ursodeoxycholic acid. Gastroenterology. 2006;130(3):715-720.

5. Marschall HU, Wagner M, Zollner G, et al. Complementary stimulation of hepatobiliary transport and detoxification systems by rifampicin and ursodeoxycholic acid in humans. Gastroenterology. 2005;129(2):476-485.

6. Ghonem NS, Boyer JL. Fibrates as adjuvant therapy for chronic cholestatic liver disease: its time has come. Hepatology. 2013;57(5):1691-1693.

7. Iwasaki S, Akisawa N, Saibara T, Onishi S. Fibrate for treatment of primary biliary cirrhosis. Hepatol Res. 2007;37 Suppl 3:S515-S517.

8. Devchand PR, Keller H, Peters JM, Vazquez M, Gonzalez FJ, Wahli W. The PPARalpha-leukotriene B4 pathway to inflammation control. Nature. 1996;384(6604):39-43.

9. Nishioka T, Hyogo H, Numata Y, et al. A nuclear receptor-mediated choleretic action of fibrates is associated with enhanced canalicular membrane fluidity and transporter activity mediating bile acid-independent bile secretion. $J$ Atheroscler Thromb. 2005;12(4):211-217.

10. Corpechot C, Chazouillères O, Poupon R. Early primary biliary cirrhosis: biochemical response to treatment and prediction of long-term outcome. J Hepatol. 2011;55(6):1361-1367. 
11. Wu D, Wu SM, Lu J, Zhou YQ, Xu L, Guo CY. Rifaximin versus nonabsorbable disaccharides for the treatment of hepatic encephalopathy: a meta-analysis. Gastroenterol Res Pract. 2013;2013:236963.

12. European Association for the Study of the Liver. EASL Clinical Practice Guidelines: management of cholestatic liver diseases. J Hepatol. 2009;51(2):237-267.

13. Nikolova D, Klingenberg SL, Gluud C. The Cochrane hepato-biliary group as a resource example of evidence-based medicine for all. Acta Med Port. 2013;26(2):81-82.

14. Kjaergard LL, Villumsen J, Gluud C. Reported methodologic quality and discrepancies between large and small randomized trials in metaanalyses. Ann Intern Med. 2001;135(11):982-989.

15. Wood L, Egger M, Gluud LL, et al. Empirical evidence of bias in treatment effect estimates in controlled trials with different interventions and outcomes: meta-epidemiological study. BMJ. 2008; 336(7644):601-605.

16. Dohmen K, Mizuta T, Nakamuta M, Shimohashi N, Ishibashi H, Yamamoto K. Fenofibrate for patients with asymptomatic primary biliary cirrhosis. World J Gastroenterol. 2004;10(6):894-898.

17. Ohira H, Sato Y, Ueno T, Sata M. Fenofibrate treatment in patients with primary biliary cirrhosis. Am J Gastroenterol. 2002;97(8):2147-2149.

18. Walker LJ, Newton J, Jones DE, Bassendine MF. Comment on biochemical response to ursodeoxycholic acid and long-term prognosis in primary biliary cirrhosis. Hepatology. 2009;49(1):337-338; author reply 338 .

19. Liberopoulos EN, Florentin M, Elisaf MS, Mikhailidis DP, Tsianos E. Fenofibrate in primary biliary cirrhosis: a pilot study. Open Cardiovasc Med J. 2010;4:120-126.

20. Levy C, Peter JA, Nelson DR, et al. Pilot study: fenofibrate for patients with primary biliary cirrhosis and an incomplete response to ursodeoxycholic acid. Aliment Pharmacol Ther. 2011;33(2):235-242.

21. Han XF, Wang QX, Liu Y, et al. Efficacy of fenofibrate in Chinese patients with primary biliary cirrhosis partially responding to ursodeoxycholic acid therapy. J Dig Dis. 2012;13(4):219-224.

22. Zhang Y, Lu J, Dai W, et al. Combination therapy of ursodeoxycholic acid and corticosteroids for primary biliary cirrhosis with features of autoimmune hepatitis: a meta-analysis. Gastroenterol Res Pract. 2013;2013:490731

23. Zhang H, Yang J, Zhu R, et al. Combination therapy of ursodeoxycholic acid and budesonide for PBC-AIH overlap syndrome: a meta-analysis. Drug Des Devel Ther. 2015;9:567-574.

24. Zhang Y, Chen K, Dai W, et al. Combination therapy of bezafibrate and ursodeoxycholic acid for primary biliary cirrhosis: A meta-analysis. Hepatol Res. 2015;45(1):48-58.
25. Farnier M, Bonnefous F, Debbas N, Irvine A. Comparative efficacy and safety of micronized fenofibrate and simvastatin in patients with primary type IIa or IIb hyperlipidemia. Arch Intern Med. 1994;154(4): $441-449$.

26. Willson TM, Brown PJ, Sternbach DD, Henke BR. The PPARs: from orphan receptors to drug discovery. J Med Chem. 2000;43(4):527-550.

27. Kok T, Bloks VW, Wolters H, et al. Peroxisome proliferator-activated receptor alpha (PPARalpha)-mediated regulation of multidrug resistance 2 (Mdr2) expression and function in mice. Biochem J. 2003;369(Pt 3): $539-547$.

28. Ros JE, Libbrecht L, Geuken M, Jansen PL, Roskams TA. High expression of MDR1, MRP1, and MRP3 in the hepatic progenitor cell compartment and hepatocytes in severe human liver disease. J Pathol. 2003;200(5):553-560.

29. Delerive P, Fruchart JC, Staels B. Peroxisome proliferator-activated receptors in inflammation control. J Endocrinol. 2001;169(3):453-459.

30. El-Sisi A, Hegazy S, El-Khateeb E. Effects of three different fibrates on intrahepatic cholestasis experimentally induced in rats. PPAR Res. 2013;2013:781348.

31. Brok J, Thorlund K, Gluud C, Wetterslev J. Trial sequential analysis reveals insufficient information size and potentially false positive results in many meta-analyses. J Clin Epidemiol. 2008;61(8):763-769.

32. Wetterslev J, Thorlund K, Brok J, Gluud C. Trial sequential analysis may establish when firm evidence is reached in cumulative meta-analysis. J Clin Epidemiol. 2008;61(1):64-75.

33. Jie L, Fan W, Weiqi D, et al. The hippo-yes association protein pathway in liver cancer. Gastroenterol Res Pract. 2013;2013:187070.

34. Prince MI, Chetwynd A, Craig WL, Metcalf JV, James OF. Asymptomatic primary biliary cirrhosis: clinical features, prognosis, and symptom progression in a large population based cohort. Gut. 2004; 53(6):865-870.

35. Zhou Y, Dai W, Lin C, et al. Protective effects of necrostatin-1 against concanavalin A-induced acute hepatic injury in mice. Mediators Inflamm. 2013;2013:706156.

36. Shen M, Chen K, Lu J, et al. Protective effect of astaxanthin on liver fibrosis through modulation of TGF- $\beta 1$ expression and autophagy. Mediators Inflamm. 2014;2014:954502.

37. Cheng P, Chen K, Xia Y, et al. Hydrogen sulfide, a potential novel drug, attenuates concanavalin A-induced hepatitis. Drug Des Devel Ther. 2014;8:1277-1286.

38. Chen K, Li J, Wang J, et al. 15-Deoxy- $\gamma$ 12,14-prostaglandin J2 reduces liver impairment in a model of ConA-induced acute hepatic inflammation by activation of PPAR $\gamma$ and reduction in NF- $\kappa \mathrm{B}$ activity. PPAR Res. 2014;2014:215631.
Drug Design, Development and Therapy

\section{Publish your work in this journal}

Drug Design, Development and Therapy is an international, peerreviewed open-access journal that spans the spectrum of drug design and development through to clinical applications. Clinical outcomes, patient safety, and programs for the development and effective, safe, and sustained use of medicines are a feature of the journal, which

\section{Dovepress}

has also been accepted for indexing on PubMed Central. The manuscript management system is completely online and includes a very quick and fair peer-review system, which is all easy to use. Visit http://www.dovepress.com/testimonials.php to read real quotes from published authors. 